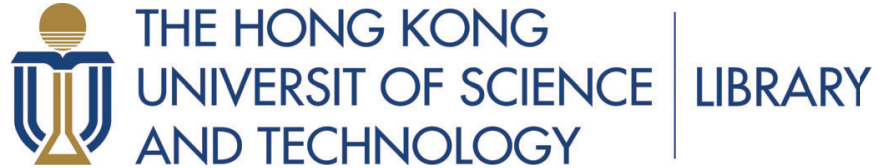

\section{HKUST SPD - INSTITUTIONAL REPOSITORY}

Title Modeling Electrochemical Impedance Spectroscopy

Authors Ciucci, Francesco

Source Current Opinion in Electrochemistry, v. 13, February 2019, p. 132-139

Version Accepted Version

DOI $\quad 10.1016 / j . c o e l e c .2018 .12 .003$

Publisher Elsevier

Copyright (C) 2019 Elsevier

License CC BY-NC-ND 4.0

This versionis availableat HKUST SPD - Institutional Repository (https://repository.ust.hk/ir)

If it is the author's pre-published version, changes introduced as a result of publishing processes such as copy-editing and formatting may not be reflected in this document. For a definitive version of this work, please refer to the published version. 


\title{
Modeling Electrochemical Impedance Spectroscopy
}

\author{
Francesco Ciucci ${ }^{\mathrm{a}, \mathrm{b}, \uparrow}$ \\ a Department of Mechanical and Aerospace Engineering, The Hong Kong University of \\ Science and Technology, Hong Kong, China \\ ${ }^{\mathrm{b}}$ Department of Chemical and Biological Engineering, The Hong Kong University of Science \\ and Technology, Hong Kong, China \\ ${ }^{\dagger}$ Corresponding author: francesco.ciucci@ust.hk
}




\begin{abstract}
Electrochemical impedance spectroscopy (EIS) is a powerful technique that is used for characterizing electrochemical systems. The EIS data can be correlated with many key physical properties, including rates of diffusion and reaction, and microstructural features. However, the EIS analysis is prone to the potential ambiguities in interpretation. Judicious modeling and its combination with statistics can be used to overcome these challenges and enhance the insight one can gain from EIS.
\end{abstract}




\section{Introduction}

Electrochemical impedance spectroscopy (EIS) is a powerful characterization technique that is widely used in fields as diverse as energy, electrocatalysis, and medicine.[1-4] Two factors make EIS particularly attractive. First, EIS data can be used to obtain physical properties, such as diffusion coefficients and chemical reaction rates, and microstructural characteristics of the electrochemical (EC) system under study.[5, 6] Second, the implementation of an EIS experiment is relatively simple.

EIS is unique among all classical EC techniques because it measures the relationship between the current and the applied potential difference in the frequency domain.[5] Specifically, the EIS technique estimates the transfer function between current and potential. To measure the EIS, one subjects an EC system to a small (ideally infinitesimal) sinusoidal voltage (potentiostatic mode) or current (galvanostatic mode) perturbation for a given set of frequencies and, concomitantly, records the resulting sinusoidal current or voltage response. From the voltage-to-current amplitude ratio and the phase lag between input and output, one obtains a complex-valued function $Z(f)$, which depends on the perturbation frequency $f$. It should be noted that, for the EIS to be meaningful, the conditions of stability, linearity, and causality need to be met. [4, 5] First, the EC system needs to be stable, because if it changes with time so will $Z(f)$. Second, it is critical that the perturbation is linear: if the amplitude of the applied sinusoidal perturbation is sufficiently small, then the response will be a sinusoid of the same frequency. Instead, if the amplitude of the sinusoidal input at frequency $f$ is large, the measured response will be the sum of sinusoids at frequencies $f, 2 f, 3 f$, etc.[7] Third, causality is required: the measured current (or voltage) must be the direct result of the voltage (or current) perturbation applied. 
Interpreting the EIS data can be challenging. In fact, in many practical cases, multiple models may be able to fit the experimental data equally well.[4] These ambiguities can be further exacerbated because some parameters of the models may not be determined with sufficient accuracy.

As schematically shown in Figure 1, we will illustrate equivalent circuit models (ECMs). We will then present physical transmission-line models, and generalize those to multiple dimensions. Following that, we will examine the distribution of relaxation times (DRT) models. Finally, we will link the EIS analysis to frequentist regression-based statistical methods and model selection.

\section{Equivalent Circuits Models}

EIS spectra are commonly analyzed using ECMs, where one uses his/her prior experience to find a circuit comprising a finite set of elementary (resistors, capacitors, and inductors) and generalized electrical circuits that can match the data. Thanks to their simplicity, ECMs are particularly attractive. However, they often are lump-parameter black-box models, which are seldom unique.

EC systems and their physical properties are typically distributed in space. In this regard, generalized impedance elements, such as the constant-phase element, the fractal element, the Gerischer element, the Warburg element, the generalized Warburg element, etc. have been developed.[4] These individual elements can be derived from rigorous physical principles and can be obtained by resolving physics-based boundary value problems. [4, 8] Phenomenological ECMs, which combine both elementary and generalized circuits in series and parallel, has been proven useful in the evaluation of the conductivities and interfacial impedances of many EC systems. [4, 9] An example related to solid-state thin-film batteries is reported in Figure 2 (a-b).[10] Selected sub-elements of the circuit in Figure 2 (a) can be used 
to model the EIS of symmetrical cells with blocking, see Figure 2 (c), or transmissive, see Figure 2 (d), electrodes. Generalized circuits have also been incorporated in software packages and are now frequently used to analyze experimental data.[4]

While the search for ECM analogs can be automated [11] using, for example, genetic [12] and gene-expression programming,[13] it is still challenging to interpret all ECMs physically and to ascertain their uniqueness. Therefore, the indiscriminate use of ECMs has been severely critiqued $[4,5,14]$ because, I quote Prof. D.D. Macdonald, "a mere development of an analog (which is now done by various computer programs) represents an incomplete analysis of the data." [1] Furthermore, the determination of the circuit parameters is mostly carried out by nonlinear regression. If the ECM has too many individual elements, circuit parameters cannot be identified with sufficient precision.[15] Also, it is not uncommon that multiple ECMs can fit the experimental EIS data equally well. For this reason, selecting only one of the plausible ECMs could prove to be a significant challenge. We will go back to these issues towards the end of this article where we will discuss the statistical analysis of the EIS data.

\section{Physical Models}

Many generalized models can be directly derived from physical principles. Resolving problemspecific physical models is significantly more challenging than constructing ad hoc ECMs. However, physical models are far more informative of the actual physics and chemistry of the EC system under study.[16] We will illustrate this point using battery and fuel cell examples.

\section{Ionic Solids}

To model the EIS of solid ionic materials one can start directly from the Poisson-Nernst-Planck (PNP) system of equations [17] equipped with suitable boundary conditions.[4] In analogy with the finite difference discretization of the linearized PNP equations, one can synthesize a 
network of infinitesimal circuits.[18] Following the pioneering work of the Maier group, the actual PNP system for mixed ionic-electronic conductors (MIECs) can be recast as a circuit with three rails.[19] A central capacitive track, representing the Poisson equation, is connected to two resistive lines, characterizing the Nernst-Plank conduction of ions and electrons, through an infinite number of capacitances. The electroneutral version of these models, where the central Poisson rail is no longer present, can be directly applied to many MIEC systems, see Figure 3. As shown in Figure 3 (a), the two transport rails for electrons (eons) and ions (ions) are connected by an infinite set of infinitesimal capacitors, which represent the so-called chemical capacitance, a quantity associated to the concentration of defects in MIECs.[20-22] The PNP framework has also been used beyond the MIEC area. To model the ionic transport in liquid electrolytes, Macdonald and coworkers employed analytical solutions [23, 24] and Moya introduced a network circuit solution method.[25, 26]

Many of these models have analytical solutions and have found application in fuel cells to determine the defect chemistry and reactivity of various MIEC materials, including the study of doped Ceria [22] cells as shown in Figure 3 (b).[20] A battery thin-film cathode is a MIEC with semi-blocking boundaries and, therefore, its EIS can also be analyzed using the same framework, see Figure 3 (c).[27] Interestingly, the circuit network can be directly linked to the microstructural characteristics of the electrode under study as shown pictorially in Figure 3 (d). [28]

\section{Porous Materials}

Analytical models stemming from physical principles are not limited to solid ionic materials but have also been used to analyze photoelectrodes,[29] dye-sensitized solar cells [30], batteries,[31-36] and fuel cells. [37-39] For complex porous EC systems, one can also mate analytical models with measured microstructural data to obtain an EIS dependent on fewer 
assumptions.[40, 41] Not only is it possible to compute the EIS of a porous electrode once its microstructure is known, but one can also leverage the measured $Z(f)$ to carry out porosimetry [5] in order to quantify the distribution of electroactive particles' sizes.[42] Using semianalytical models, one can obtain the probability distribution function of the electroactive particles' radii $[43,44]$ as well as the electrode tortuosity.[45] The porous materials' EIS models need not to be necessarily analytical but can also be generated from computational boundary value problems, as recently shown by Drummond and co-workers [46], who have synthesized physically rigorous circuit networks using Chebyshev interpolation.[47] This numerical approach, unlike finite differencing, ensures spectral convergence,[48] implying that fewer unknowns are needed and that the numerical solution is, in principle, closer to the exact one for a broader range of frequencies.

\section{Multidimensional Models}

Circuit models have useful graphical representation, but results that are more general can be obtained by resolving the underlying partial differential equations in multiple dimensions.[4951] In this context, one can overcome the geometrical assumptions needed in 1D by assessing the impact of the EC system geometry, including the microstructure [51] and placement [5255] of the electrodes, on the EIS. Furthermore, a typical EIS measurement spans frequencies within several orders of magnitude. Therefore, mesh optimization is critical. Luckily, solving partial differential equations can leverage modern computational science to enable the control of the numerical error by adapting the mesh a posteriori $[56,57]$. One potentially intriguing approach is to use optimized goal-oriented strategies for mesh adaptivity.[58]

\section{Distribution of Relaxation, Diffusion, and Capacitance Times}

The analysis of EIS data using the DRT has recently attracted considerable research interest as shown in two recent reviews.[59, 60] The DRT aims at estimating the relaxation 
characteristics of an EC system and relies on the premise that, if we subject an EC system to a small step perturbation (e.g. a current impulse), the response (e.g. the voltage) will decay exponentially with a particular distribution of timescales. [60, 61] It is therefore natural to model the $Z(f)$ as follows (see $[60,61]$ for details):

$$
Z(f)=R_{0}+i 2 \pi f L_{0}+\int_{0}^{\infty} \frac{g(\tau)}{1+i 2 \pi f \tau} d \tau
$$

where $R_{0}$ is an ohmic resistance, $L_{0}$ is an inductance, and $g(\tau)$ is a timescale distribution.[62] Since the frequencies of EIS experiments are typically spaced logarithmically, (1) can be conveniently rewritten as

$$
Z(f)=R_{0}+i 2 \pi f L_{0}+\int_{-\infty}^{\infty} \frac{\gamma(\ln \tau)}{1+i 2 \pi f \tau} d \ln \tau
$$

Estimating the function $\gamma(\ln \tau)=\tau g(\tau)$ provides a direct access to the time characteristics of the EC system and can guide its physical analysis. [59, 62] Notably, this approach has been applied to the selection of models for solid oxide fuel cells [63-67] and lithium ion batteries. $[68,69]$ An example is shown in Figure 4 (a-c). The EIS data reported in Figure 4 (a) were first collected from a $\mathrm{LiFePO}_{4}$ half-cell with a state of charge ranging from 10 to $100 \%$ and then processed with DRT, Figure 4 (b). The identification of peaks evolving with the charge state of the battery could be finally linked to an ECM featuring elementary and generalized circuits, see Figure 4 (c). [70]

It must be noted, however, that deconvolving $\gamma(\ln \tau)$ from (2) is not a trivial task because the underlying problem is ill-posed. In this regard, many methods have been developed and leverage either the Fourier transform [71], maximum entropy [72], Monte Carlo sampling [73], genetic programming [74], operatorial calculus, [75] or ridge regression (RR).[76, 77] Among the various options, two factors make RR particularly appealing. First, the RR problem can be recast as a constrained quadratic programming problem, whose unique solution can be readily 
computed. Second, thanks to the link between RR and Bayesian statistics, [78] one can 1) estimate the credibility of $\gamma(\ln \tau)$, providing probability bands, see Figure 4 (d); and 2) rationally optimize the regression process to detect discontinuities in the DRT or even corrupted EIS data. $[61,79]$

Technically, the DRT method can only be applied under the hypothesis that $|Z(f)|$ is bounded. In turn, this makes the DRT unsuitable for certain applications. Fortunately, there have been two important extensions of the DRT methods. One approach pioneered by the Ivers-Tiffée and Tsur groups $[80,81]$ consists in transforming the experimental $Z(f)$ in capacitive form by computing the complex capacitance $C(f)$ as follows:

$$
C(f)=\frac{1}{i 2 \pi f Z(f)}
$$

The distribution of differential capacitance can then be estimated by deconvolving $\gamma(\ln \tau)$ with the model $C(f)=\int_{-\infty}^{\infty} \frac{\gamma(\ln \tau)}{1+i 2 \pi f \tau} d \ln \tau$. Using a completely different approach, Song and Bazant [6] recently reinterpreted porosimetry in a nonparametric fashion by modeling the EIS as the parallel distribution of micro-level circuits.[82] Mathematically, this corresponds to using the following model

$$
Y(f)=\frac{1}{Z(f)}=\int_{0}^{\infty} \frac{P(\tau)}{z_{D}(f, \tau)} d \tau
$$

where $P(\tau)$ is the distribution of diffusion times (DDT) and $z_{D}(f, \tau)$ is the impedance of an individual finite-length model.[82] The DDT gives the diffusional time characteristics of complex electrodes, providing a proxy for the electrode particle distribution. An example is given for the EIS data shown in Figure 4 (e); the modified DDT $q\left(\ln \frac{\tau}{s}\right)=\tau P(\tau)$ is obtained using RR on the model (4), see Figure 4 (f). [6]

\section{Statistical Analysis}


The fitting of the EIS data is almost invariably done using frequentist statistics. Leveraging regression, one searches the set of parameters $\boldsymbol{\theta}=\left[\begin{array}{llll}\theta_{1} & \theta_{2} & \ldots & \theta_{M}\end{array}\right]^{T}$ (note $M \ll N$ ) of a given model that best fit the data by minimizing the following objective function

$$
S(\boldsymbol{\theta})=\sum_{n=1}^{N} \frac{\left|Z^{\mathrm{exp}}\left(f_{n}\right)-Z^{\text {model }}\left(f_{n}, \boldsymbol{\theta}\right)\right|^{2}}{\sigma_{n}^{2}}
$$

where $N$ is the total number of measurements, $Z^{\exp }\left(f_{n}\right)$ is the experimental impedance measured at a frequency $f_{n}, Z^{\text {model }}\left(f_{n}, \boldsymbol{\theta}\right)$ is the model, and $\sigma_{n}$ is the standard deviation of the $n^{\text {th }}$ measurement. The estimated value of the vector of parameters, $\boldsymbol{\theta}$, is given by $\widehat{\boldsymbol{\theta}}=$ $\underset{\boldsymbol{\theta}}{\operatorname{argmin}} S(\boldsymbol{\theta})$. However, the analysis cannot stop at predicting $\widehat{\boldsymbol{\theta}}$. The quality of the regression should be assessed quantitatively by comparing the Nyquist representation of the EIS data and the fitted model.[8] Following that, it is often suggested to carry out a $\chi^{2}$ test.[8] Additionally, to obtain $\widehat{\boldsymbol{\theta}} \pm \Delta \boldsymbol{\theta}$, one needs to estimate the confidence intervals at $\widehat{\boldsymbol{\theta}}$. One strategy is to use the asymptotic variance-covariance matrix, which gives approximated confidence ellipsoids as schematically illustrated in Figure 1.[15, 83] This matrix is closely related to the sensitivity of $Z^{\text {model }}\left(f_{n}, \boldsymbol{\theta}\right)$ with respect to $\boldsymbol{\theta}$. Loosely speaking, the estimated uncertainty, $\Delta \boldsymbol{\theta}$, is largest for parameters that are least sensitive, that is for those where $\frac{\partial Z^{\text {model }}\left(f_{n}, \boldsymbol{\theta}\right)}{\partial \theta_{m}}($ with $m=1, \ldots, M)$ is the smallest, or the most correlated. $[15,83]$ To measure the statistics of the estimation process and its nonlinearities, one can conduct bootstrapping (i.e. random sampling with replacement) and produce a collection of synthetic experiments, and from each of those regression can be performed, leading to a distribution of estimated $\widehat{\boldsymbol{\theta}} \cdot[84,85]$ As shown in Figure 1, these values can deviate from the asymptotic confidence regions bound by the asymptotic ellipsoids unless certain conditions (small errors and limited nonlinearities) are met. 
The discussion above assumes that a single exact model exists. In general, this is not the case, as the physics of an EC system is often not known a priori. It is clear that, if multiple plausible models are available, one should be able to rank them. Luckily, there are criteria for model selection. A notable example is the Akaike information criterion (AIC).[86] The AIC estimates the quality of the statistical model for given data by computing [87]

$$
\mathrm{AIC}=\ell(\widehat{\boldsymbol{\theta}})-\operatorname{dim} \widehat{\boldsymbol{\theta}}
$$

where $\ell(\widehat{\boldsymbol{\theta}})$ is the log-likelihood of the model at the estimated $\widehat{\boldsymbol{\theta}}$ and $\operatorname{dim} \widehat{\boldsymbol{\theta}}=M$. In layman's terms, the AIC can be thought as the difference between the goodness of fit $(\ell(\widehat{\boldsymbol{\theta}}))$, which increases with the number $(M)$ of parameters available, and the model complexity defined as the total number of model parameters $(\operatorname{dim} \widehat{\boldsymbol{\theta}}=M)$. The best model over various options is obtained by maximizing the AIC.

\section{Concluding Remarks}

The ambiguity of interpretation is perhaps the biggest challenge facing the EIS technique. Modeling can play a central role in resolving this problem. To this end, it is critical to combine the various modeling approaches illustrated above. Model selection should comply with information-maximizing criteria and be inspired by physics to allow for enhanced insight. It is also expected that statistics, beyond mere regression of a single spectrum, will be increasingly crucial for the analysis of EIS data. For example, creating and analyzing large datasets $[88,89]$ could pave the way for significant advancements in the field. 


\section{Acknowledgments}

Francesco Ciucci gratefully acknowledges the Research Grants Council of Hong Kong for support through the projects 16207615, 16227016, and 16204517. The authors also acknowledge the support from the Guangzhou Science and Technology Program (No.201807010074). Francesco Ciucci thanks Mr. M.B. Effat and Mr. T.H. Wan for their critical feedback on the article draft. 


\section{List of Abbreviations and Symbols}

DDT: Distribution of Diffusion Times

DRT: Distribution of Relaxation Times

EC: Electrochemical

ECM: Equivalent Circuit Model

EIS: Electrochemical Impedance Spectroscopy

MIEC: Mixed Ionic Electronic Conductor

PNP: Poisson-Nernst-Planck

RR: Ridge Regression

$C(f)$ : Complex Capacitance

$\operatorname{dim} \boldsymbol{\theta}$ : Number of EIS Model Parameters

$f$ : Frequency

$g(\tau):$ Distribution of Relaxation Times

$\gamma(\ln \tau):$ Modified Distribution of Relaxation Times

$\ell(\boldsymbol{\theta}):$ Log Likelihood

$\tau$ : Timescale

$\boldsymbol{\theta}$ : Set of EIS Model Parameters 
$\widehat{\boldsymbol{\theta}}$ : Estimated Parameter

$\Delta \boldsymbol{\theta}$ : Uncertainty on the Estimated Parameters

$Z(f)$ : Impedance 
$\underline{\text { References: }}$

[1] D.D. Macdonald, Electrochimica Acta, 51 (2006) 1376-1388.

[2] E.P. Randviir, C.E. Banks, Analytical Methods, 5 (2013) 1098-1115.

[3] G.M. Rupp, A.K. Opitz, A. Nenning, A. Limbeck, J. Fleig, Nature Materials, 16 (2017) 640.

[4] E. Barsoukov, J.R. Macdonald, Impedance Spectroscopy: Theory, Experiment, and Applications, $3^{\text {rd }}$ ed., Wiley, Hoboken, NJ, 2018.

[5] A. Lasia, Electrochemical Impedance Spectroscopy and its Applications, Springer, New York, 2014.

[6] J. Song, M.Z. Bazant, Physical Review Letters, 120 (2018) 116001.

[7] F. Fasmin, R. Srinivasan, Journal of the Electrochemical Society, 164 (2017) H443-H455.

[8] M.E. Orazem, B. Tribollet, Electrochemical Impedance Spectroscopy, $2^{\text {nd }}$ ed., Wiley, Hoboken, N.J., 2017.

[9] J.E.B. Randles, Discussions of the Faraday Society, 1 (1947) 11-19.

[10] Y. Iriyama, T. Kako, C. Yada, T. Abe, Z. Ogumi, Solid State Ionics, 176 (2005) 23712376.

[11] P.M. Ramos, F.M. Janeiro, Measurement, 46 (2013) 4379-4387.

[12] J.R. Koza, Statistics and Computing, 4 (1994) 87-112.

[13] C. Ferreira, Complex Systems, 13 (2001) 87-129.

[14] I. Lelidis, G. Barbero, Physical Review E, 95 (2017) 052604.

[15] F. Ciucci, Electrochimica Acta, 87 (2013) 532-545.

[16] A. Nenning, A.K. Opitz, T.M. Huber, J. Fleig, Physical Chemistry Chemical Physics, 16 (2014) 22321-22336.

[17] J. Maier, Physical Chemistry of Ionic Materials: Ions and Electrons in Solids, Wiley, Chichester, England, 2004.

[18] C.T. Sah, Solid-State Electronics, 13 (1970) 1547-1575.

[19] J. Jamnik, J. Maier, Physical Chemistry Chemical Physics, 3 (2001) 1668-1678.

[20] W. Lai, S.M. Haile, Journal of the American Ceramic Society, 88 (2005) 2979-2997.

[21] W. Lai, S.M. Haile, Physical Chemistry Chemical Physics, 10 (2008) 865-883.

[22] D. Chen, S.R. Bishop, H.L. Tuller, Chemistry of Materials, 26 (2014) 6622-6627.

[23] J.R. Macdonald, D.R. Franceschetti, The Journal of Chemical Physics, 68 (1978) 16141637.

[24] L.R. Evangelista, E.K. Lenzi, G. Barbero, J.R. Macdonald, Journal of Physics: Condensed Matter, 23 (2011) 485005.

[25] J. Horno, A.A. Moya, C.F. González-Fernández, Journal of Electroanalytical Chemistry, 402 (1996) 73-80.

[26] A.A. Moya, J. Horno, The Journal of Physical Chemistry B, 103 (1999) 10791-10799.

[27] J. Jamnik, Solid State Ionics, 157 (2003) 19-28.

[28] C. Zhu, R.E. Usiskin, Y. Yu, J. Maier, Science, 358 (2017).

[29] J. Bisquert, The Journal of Physical Chemistry B, 106 (2002) 325-333.

[30] F. Fabregat-Santiago, J. Bisquert, G. Garcia-Belmonte, G. Boschloo, A. Hagfeldt, Solar Energy Materials and Solar Cells, 87 (2005) 117-131.

[31] M. Doyle, J.P. Meyers, J. Newman, Journal of the Electrochemical Society, 147 (2000) 99-110.

[32] J.P. Meyers, M. Doyle, R.M. Darling, J. Newman, Journal of the Electrochemical Society, 147 (2000) 2930-2940.

[33] G. Sikha, R.E. White, Journal of the Electrochemical Society, 154 (2007) A43-A54.

[34] G. Sikha, R.E. White, Journal of the Electrochemical Society, 155 (2008) A893-A902.

[35] U. Tröltzsch, O. Kanoun, H.-R. Tränkler, Electrochimica Acta, 51 (2006) 1664-1672. 
[36] M.D. Murbach, D.T. Schwartz, Journal of the Electrochemical Society, 165 (2018) A297A304.

[37] H. Zhu, R.J. Kee, Journal of the Electrochemical Society, 153 (2006) A1765-A1772.

[38] H. Nara, S. Tominaka, T. Momma, T. Osaka, Journal of the Electrochemical Society, 158 (2011) B1184-B1191.

[39] Y. Fu, S. Poizeau, A. Bertei, C. Qi, A. Mohanram, J.D. Pietras, M.Z. Bazant, Electrochimica Acta, 159 (2015) 71-80.

[40] C.-F. Chen, P.P. Mukherjee, Physical Chemistry Chemical Physics, 17 (2015) 9812-9827. [41] S. Cho, C.-F. Chen, P.P. Mukherjee, Journal of the Electrochemical Society, 162 (2015) A1202-A1214.

[42] M. Musiani, M. Orazem, B. Tribollet, V. Vivier, Electrochimica Acta, 56 (2011) 80148022 .

[43] J. Song, M.Z. Bazant, Journal of the Electrochemical Society, 160 (2013) A15-A24.

[44] J. Song, M.Z. Bazant, Electrochimica Acta, 131 (2014) 214-227.

[45] Y. Fu, Y. Jiang, S. Poizeau, A. Dutta, A. Mohanram, J.D. Pietras, M.Z. Bazant, Journal of the Electrochemical Society, 162 (2015) F613-F621.

[46] R. Drummond, S. Zhao, D.A. Howey, S.R. Duncan, Journal of Energy Storage, 10 (2017) 48-55.

[47] R. Drummond, D.A. Howey, S.R. Duncan, Journal of Power Sources, 277 (2015) 317328.

[48] L.N. Trefethen, Approximation Theory and Approximation Practice, Society for Industrial and Applied Mathematics, 2012.

[49] F. Ciucci, W.C. Chueh, D.G. Goodwin, S.M. Haile, Physical Chemistry Chemical Physics, 13 (2011) 2121-2135.

[50] C. Bao, W.G. Bessler, Journal of Power Sources, 278 (2015) 675-682.

[51] S.J. Cooper, A. Bertei, D.P. Finegan, N.P. Brandon, Electrochimica Acta, 251 (2017) 681 689.

[52] J. Fleig, S. Rodewald, J. Maier, Journal of Applied Physics, 87 (2000) 2372-2381.

[53] C. Chen, D. Chen, W.C. Chueh, F. Ciucci, Physical Chemistry Chemical Physics, 16 (2014) 11573-11583.

[54] B.A. Boukamp, N. Hildenbrand, H.J.M. Bouwmeester, D.H.A. Blank, Solid State Ionics, 283 (2015) 81-90.

[55] A. Bertei, G. Arcolini, J.P. Ouweltjes, Z. Wuillemin, P. Piccardo, C. Nicolella, Electrochimica Acta, 208 (2016) 129-141.

[56] F. Ciucci, Y. Hao, D.G. Goodwin, Physical Chemistry Chemical Physics, 11 (2009) 11243-11257.

[57] J. Liu, F. Ciucci, Physical Chemistry Chemical Physics, 19 (2017) 26310-26321.

[58] R. Becker, R. Rannacher, Acta Numerica, 10 (2003) 1-102.

[59] E. Ivers Tiffée, A. Weber, Journal of the Ceramic Society of Japan, 125 (2017) 193-201.

[60] K. Kobayashi, T.S. Suzuki, Journal of the Physical Society of Japan, 87 (2018) 094002.

[61] F. Ciucci, C. Chen, Electrochimica Acta, 167 (2015) 439-454.

[62] A. Leonide, V. Sonn, A. Weber, E. Ivers-Tiffée, Journal of the Electrochemical Society, 155 (2008) B36-B41.

[63] H. Schichlein, A.C. Müller, M. Voigts, A. Krügel, E. Ivers-Tiffée, Journal of Applied Electrochemistry, 32 (2002) 875-882.

[64] V. Sonn, A. Leonide, E. Ivers-Tiffée, Journal of the Electrochemical Society, 155 (2008) B675.

[65] A. Bertei, E. Ruiz-Trejo, F. Tariq, V. Yufit, A. Atkinson, N.P. Brandon, International Journal of Hydrogen Energy, 41 (2016) 22381-22393.

[66] B.A. Boukamp, A. Rolle, Solid State Ionics, 314 (2018) 103-111. 
[67] H.G. Seo, Y. Choi, W. Jung, Advanced Energy Materials, 8 (2018) 1703647.

[68] J.P. Schmidt, T. Chrobak, M. Ender, J. Illig, D. Klotz, E. Ivers-Tiffée, Journal of Power Sources, 196 (2011) 5342-5348.

[69] J.P. Schmidt, P. Berg, M. Schönleber, A. Weber, E. Ivers-Tiffée, Journal of Power Sources, 221 (2013) 70-77.

[70] J. Illig, M. Ender, T. Chrobak, J.P. Schmidt, D. Klotz, E. Ivers-Tiffée, Journal of the Electrochemical Society, 159 (2012) A952-A960.

[71] B.A. Boukamp, Electrochimica Acta, 154 (2015) 35-46.

[72] T. Horlin, Solid State Ionics 107 (1998) 241-253.

[73] E. Tuncer, S.M. Gubanski, IEEE Transactions on Dielectrics and Electrical Insulation, 8 (2001).

[74] A.B. Tesler, D.R. Lewin, S. Baltianski, Y. Tsur, Journal of Electroceramics, 24 (2009) 245-260.

[75] M. Žic, S. Pereverzyev Jr., RICAM-Report 16 (2018).

[76] M. Saccoccio, T.H. Wan, C. Chen, F. Ciucci, Electrochimica Acta, 147 (2014) 470-482.

[77] T.H. Wan, M. Saccoccio, C. Chen, F. Ciucci, Electrochimica Acta, 184 (2015) 483-499.

[78] D. Calvetti, E. Somersalo, Inverse Problems, 24 (2008) 034013.

[79] M.B. Effat, F. Ciucci, Electrochimica Acta, 247 (2017) 1117-1129.

[80] A. Oz, S. Hershkovitz, N. Belman, E. Tal-Gutelmacher, Y. Tsur, Solid State Ionics, 288 (2016) 311-314.

[81] M. Schönleber, E. Ivers-Tiffée, Electrochemistry Communications, 61 (2015) 45-48.

[82] W. Lai, F. Ciucci, Journal of the Electrochemical Society, 158 (2011) A115-A121.

[83] F. Ciucci, T. Carraro, W.C. Chueh, W. Lai, Electrochimica Acta, 56 (2011) 5416-5434.

[84] M.A. Abud Kappel, F.C. Peixoto, G.M. Platt, R.P. Domingos, I.N. Bastos, Applied Soft Computing, 50 (2017) 183-193.

[85] B. Efron, T. Hastie, Cambridge University Press, Cambridge, 2016.

[86] H. Akaike, IEEE Transactions on Automatic Control, 19 (1974) 716-723.

[87] K.P. Burnham, K.P. Burnham, D.R. Anderson, Model Selection and Multimodel Inference: A Practical Information-Theoretic Approach, Springer, New York, NY, 2002.

[88] A.S. Bondarenko, Analytica Chimica Acta, 743 (2012) 41-50.

[89] M.D. Murbach, D.T. Schwartz, Journal of the Electrochemical Society, 164 (2017) E3311E3320. 


\section{References Selected and Annotated as being Papers of Special}

\section{Interest}

** J. Song, M.Z. Bazant, Physical Review Letters, 120 (2018) 116001.

DDT is an innovative approach to analyze unbounded impedance data by elegantly linking the EIS to the distribution of diffusive microstructural timescales.

** J. Jamnik, J. Maier, Physical Chemistry Chemical Physics, 3 (2001) 1668-1678.

The PNP equations can be recast as a transmission-line model and further simplified following electroneutrality to obtain directly quantities directly observable by experiments..

** W. Lai, S.M. Haile, Journal of the American Ceramic Society, 88 (2005) 2979-2997.

The defect chemistry of Ceria is obtained from a model-based analysis of the EIS data obtained for a wide span of oxygen partial pressures and temperatures.

**F. Ciucci, C. Chen, Electrochimica Acta, 167 (2015) 439-454.

The DRT has a deep connection to Bayesian statistics. The DRT is a probability distribution function whose deconvolution can be optimized.

** J. Illig, M. Ender, T. Chrobak, J.P. Schmidt, D. Klotz, E. Ivers-Tiffée, Journal of The Electrochemical Society, 159 (2012) A952-A960.

DRT-inspired ECM leads to disentangling process-limiting factors.

* A. Oz, S. Hershkovitz, N. Belman, E. Tal-Gutelmacher, Y. Tsur, Solid State Ionics, 288 (2016) 311-314.

The complex capacitance is used to estimate the distribution of timescales in supercapacitors overcoming the limitations of the DRT.

*M. Schönleber, E. Ivers-Tiffée, Electrochemistry Communications, 61 (2015) 45-48.

The complex capacitance can lead to the determination of the distribution of timescales in batteries.

* R. Drummond, S. Zhao, D.A. Howey, S.R. Duncan, Journal of Energy Storage, 10 (2017) 48-55.

Equivalent circuits can be synthesized from partial differential equations directly.

* S.J. Cooper, A. Bertei, D.P. Finegan, N.P. Brandon, Electrochimica Acta, 251 (2017) 681689.

Homogenization can be used to upscale EIS of microstructures to the macroscopic level. 
Impedance

\section{Modeling}

Equivalent Circuit Analogs

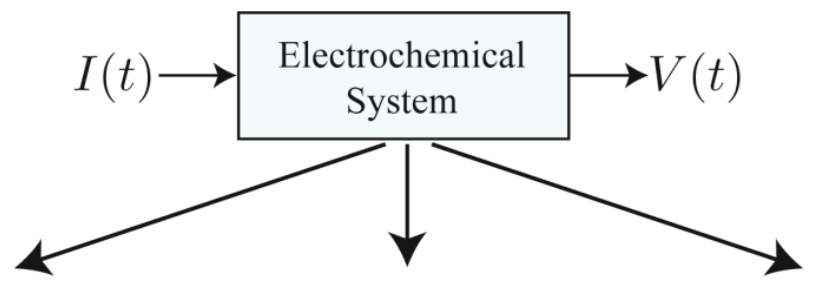

Physical Models

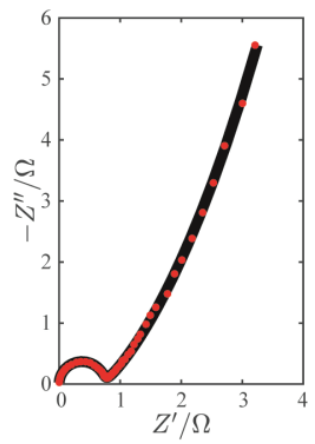

Distribution of Timescales

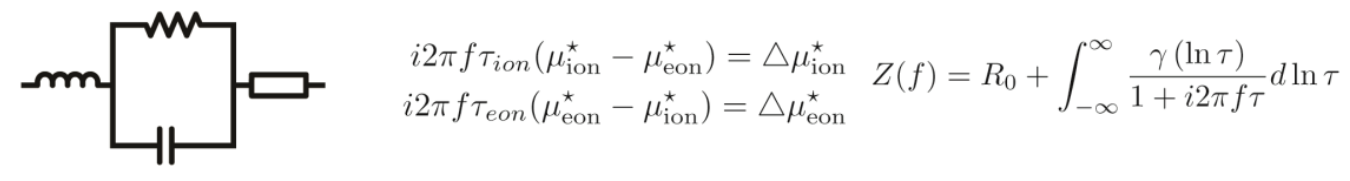

\section{Analysis}

Regression:

$$
\begin{aligned}
\hat{\boldsymbol{\theta}} & =\arg \min _{\boldsymbol{\theta}} S(\boldsymbol{\theta}) \\
S(\boldsymbol{\theta}) & =\sum_{j=1}^{N} \frac{\left|Z_{\mathrm{exp}}\left(f_{j}\right)-Z_{\text {model }}\left(f_{j}, \boldsymbol{\theta}\right)\right|^{2}}{\sigma_{j}^{2}}
\end{aligned}
$$

Akaike Information Criterion:

$$
\mathrm{AIC}=\ell(\hat{\boldsymbol{\theta}})-\operatorname{dim}(\hat{\boldsymbol{\theta}})
$$

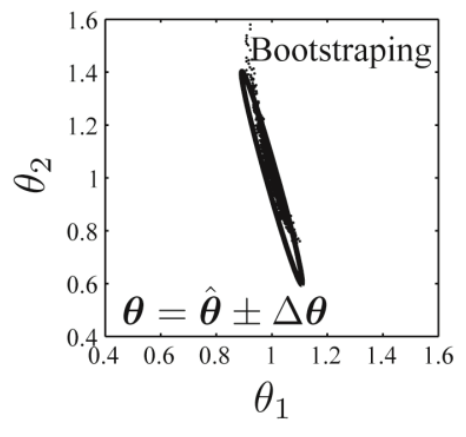

Figure 1 - Schematic illustration of the EIS modeling tools covered in this review, including ECMs, physical partial-differential-equation-based models, and distribution of timescales. Statistical analysis is also briefly discussed and covers regression and bootstrapping. 
(a)

\begin{tabular}{|l|l|l|}
\hline $\mathrm{Li}$ & $\mathrm{LiPON}$ & $\mathrm{LiCoO}_{2}$ \\
\hline
\end{tabular}

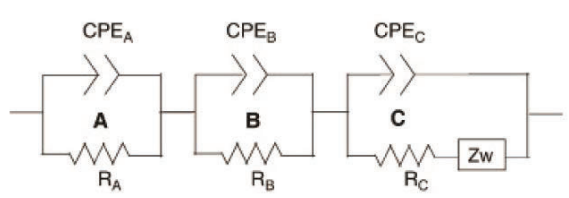

(c)

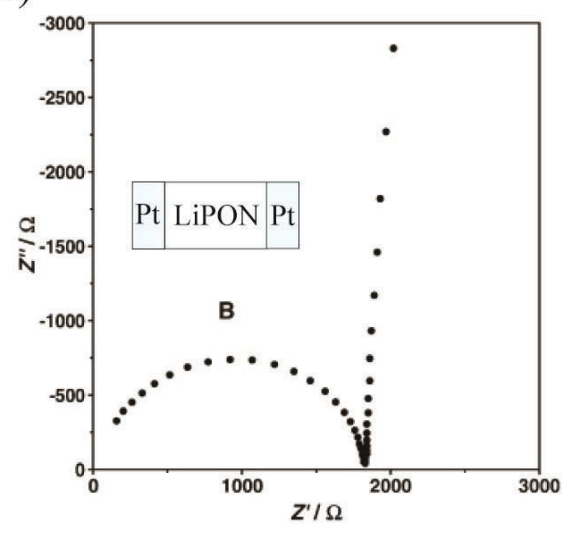

(b)

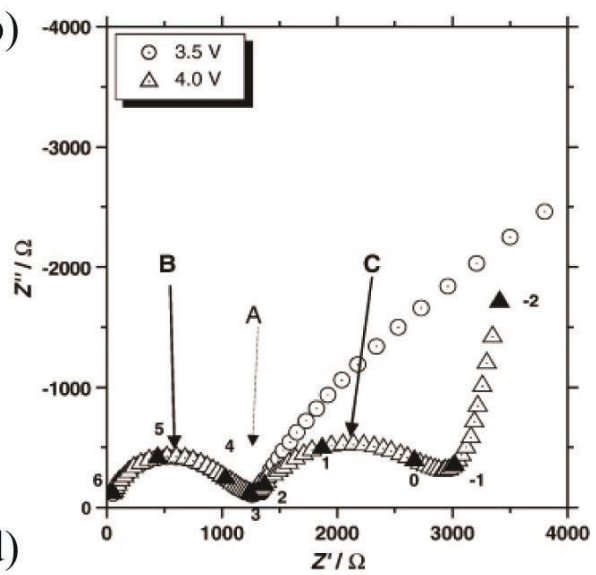

(d)

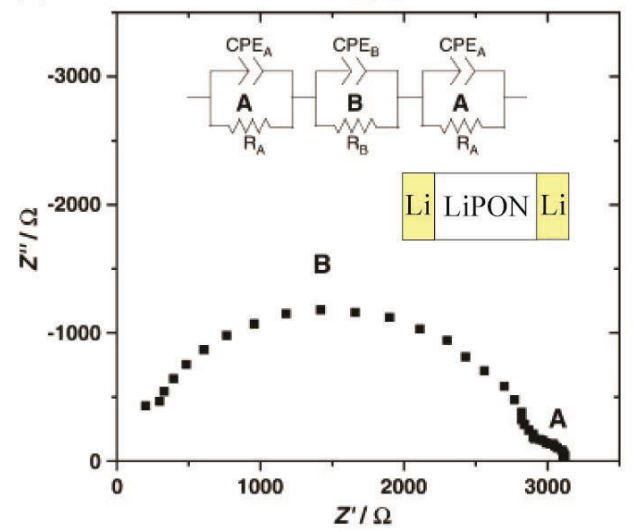

Figure 2 - (a) Phenomenological ECM of a solid-state thin-film battery in the Li/LiPON (lithium phosphorus oxynitride glass electrolyte)/ $\mathrm{LiCoO}_{2}$ configuration, where the subcircuits labeled as $\mathrm{A}, \mathrm{B}$, and $\mathrm{C}$, correspond to the impedances of the $\mathrm{Li} \mid \mathrm{LiPON}$ interface, the bulk Li transport of $\mathrm{LiPON}$, and the $\mathrm{LiPON} \mid \mathrm{LiCO}_{2}$ interface $+\mathrm{LiCO}_{2}$ electrode, respectively. (b) Impedances of the $\mathrm{Li} / \mathrm{LiPON} / \mathrm{LiCoO}_{2}$ battery at 3.5 and $4 \mathrm{~V}$. Nyquist plots of the EIS of symmetrical (c) $\mathrm{Li} / \mathrm{LiPON} / \mathrm{Li}$ (transmissive electrode) and (d) Pt/LiPON/Pt (blocking electrode) cells. Reproduced with permission from ref. [10]. 
(a)
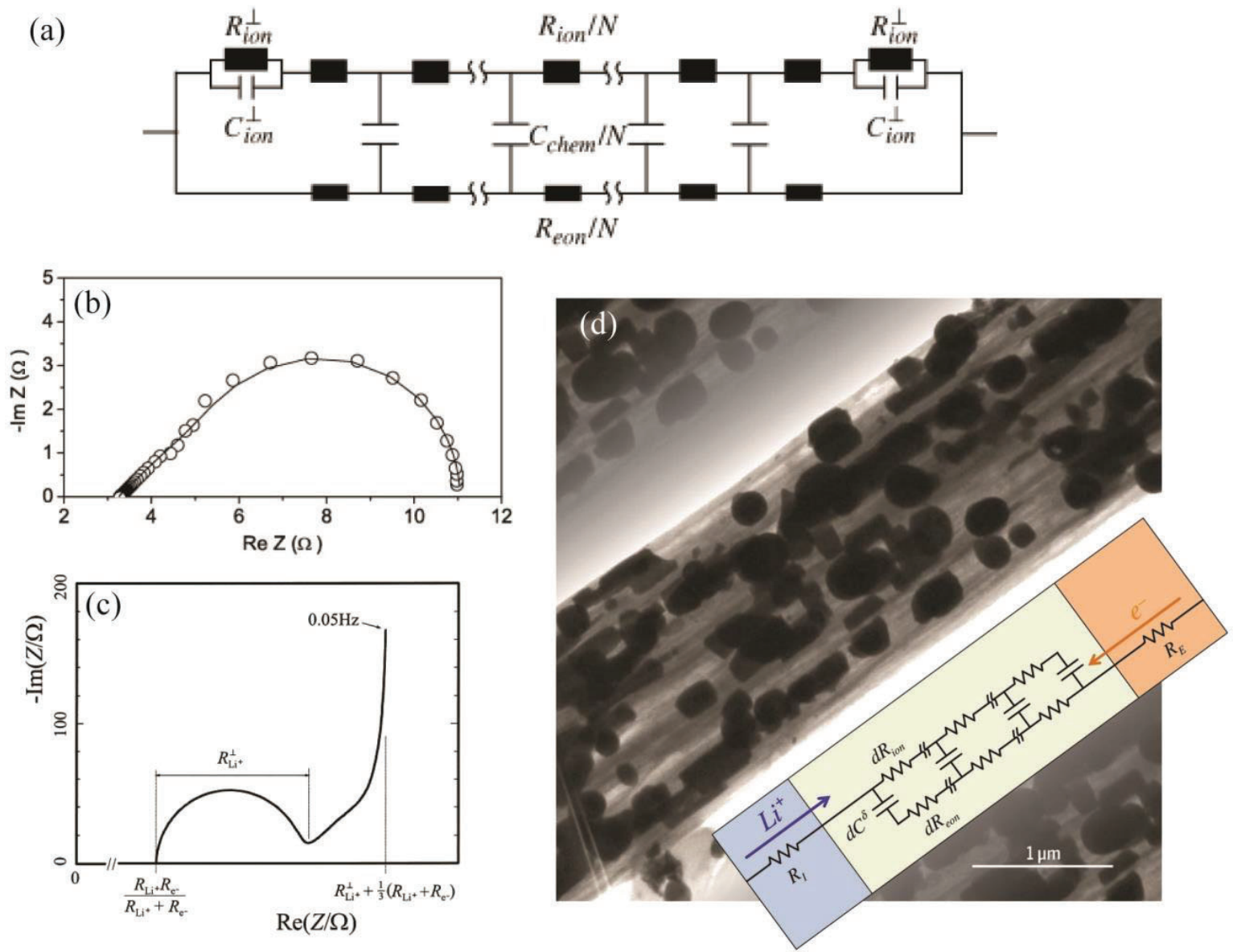

Figure 3 - (a) PNP-derived transmission line model for a slab of doped ceria with Pt currentcollecting leads and (b) corresponding Nyquist representation of the EIS. Reproduced with permission from [20], Wiley-VCH. (c) EIS impedance of a battery cathode thin film. Reproduce with permission from ref. [27]. (d) Representative battery nanostructure and circuit network. Reproduced with permission from ref. [28], American Association for the Advancement of Science. 
(a)

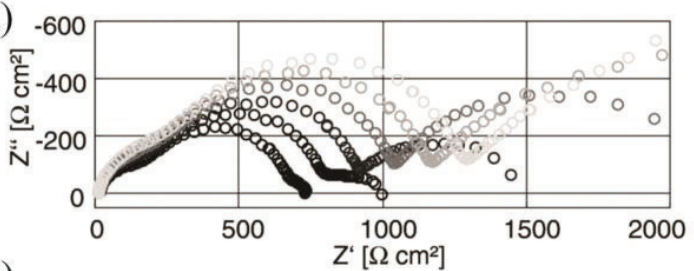

(b)

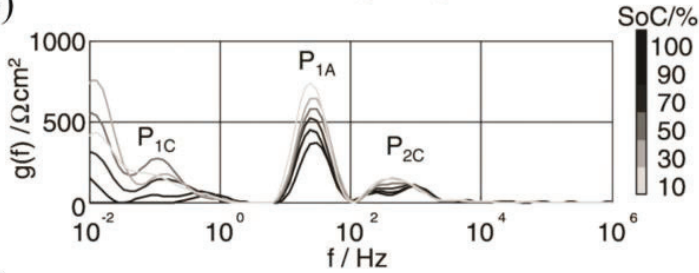

(c)

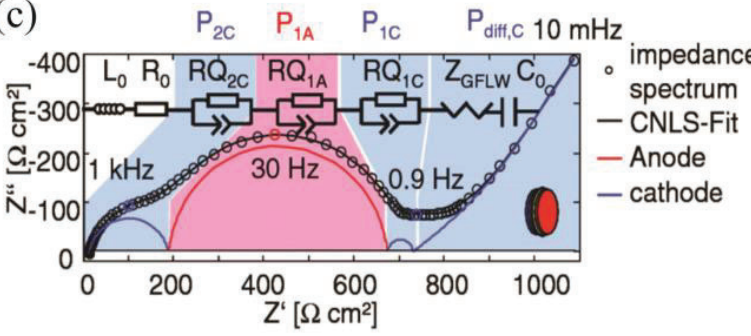

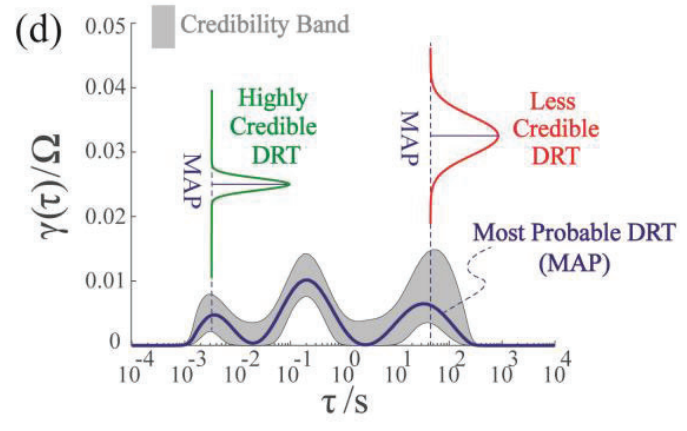

(e)

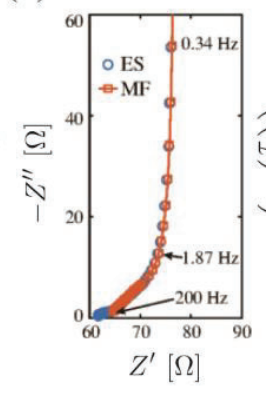

(f)

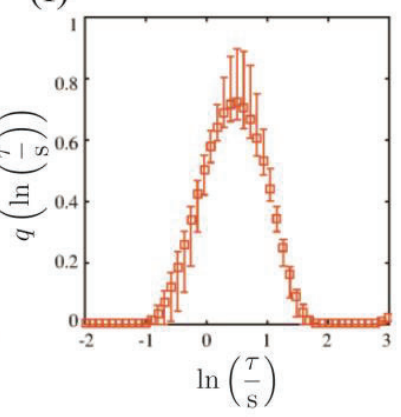

Figure 4 - (a) EIS of a $\mathrm{LiFePO}_{4}$ half-cell measured at various state of charge, (b) the corresponding DRT of the processed EIS, and (c) the ECM formulated using the DRT analysis. Reproduced with permission from ref. [70], Electrochemical Society. (d) DRT obtained from RR and illustration of the credibility intervals. Reproduced with permission from ref. [79]. (e) Experimental spectra (ES) of a carbon nanotube electrode with the model fit (MF) and (f) the corresponding DDT with $q\left(\ln \frac{\tau}{s}\right)=\tau P(\tau)$. Reproduced with permission from ref. [6], American Physical Society. 